\title{
Spondyloepimetaphyseal dysplasia, PAPSS2 type
}

INSERM

\section{Source}

INSERM. (1999). Orphanet: an online rare disease and orphan drug data base.

Spondyloepimetaphyseal dysplasia, PAPSS2 type. ORPHA:93282

Spondyloepimetaphyseal dysplasia (SEMD), Pakistani type is characterized by short

stature, short and bowed lower limbs, mild brachydactyly, kyphoscoliosis, abnormal gait, enlarged knee joints, precocious osteoarthropathy, and normal intelligence. 